Check for updates

Cite this: Chem. Commun., 2021, 57,5810

Received 28th February 2021 Accepted 10th May 2021

DOI: $10.1039 / \mathrm{d} 1 \mathrm{cc} 01110 \mathrm{c}$

rsc.li/chemcomm

\section{A cysteine-selective fluorescent probe for monitoring stress response cysteine fluctuations $\uparrow$}

\author{
Yang Yang, ${ }^{\text {ab }}$ Liangwei Zhang, (D)*b Xia Zhang, ${ }^{\mathrm{b}}$ Shudi Liu, ${ }^{\mathrm{c}}$ Yue Wang, ${ }^{\mathrm{b}}$ Li Zhang, ${ }^{\mathrm{b}}$ \\ Zhuo Ma, ${ }^{c}$ Huiyan You*a and Lingxin Chen (D)*bd
}

Rare studies provided evidence for the real-time monitoring of stress response cysteine fluctuations. Here, we have successfully designed and synthesized a cysteine-selective fluorescent probe 1 to monitor stress response Cys fluctuations, providing visual evidence of $\mathrm{Hg}^{2+}$ regulated cysteine fluctuations for the first time, which may open a new way to help researchers to reveal the mechanism of heavy metal ion poisoning.

Biological thiols, indispensable reactive sulfur species (RSS) in the body, are present in the form of low molecular weight thiols or protein thiols, mainly including cysteine (Cys), glutathione (GSH), homocysteine (Hcy) and cysteine residues in proteins. And they can transform into different oxidative intermediates when suffering from intracellular or external environmental changes. They play important roles in various physiological processes, such as maintaining redox balance, protein synthesis and detoxification. ${ }^{1}$ Many studies have suggested that the endogenous concentration changes of these thiols were associated with the functional status of the corresponding enzymes and proteins. Their abnormal levels are related to diseases. ${ }^{2-5}$ Hcy can be catalyzed by cystathionine $\beta$-synthase (CBS) to condense with serine to form cystathionine, which is then hydrolyzed to Cys by cystathionine $\gamma$-lyase (CSE). Cysteine is one of the natural components that make up glutathione (GSH). ${ }^{6}$ It is a non-essential amino acid for the human body and mainly exists in proteins. Abnormal levels of cysteine

\footnotetext{
${ }^{a}$ College of Environment and Chemical Engineering, Dalian University, Dalian 116622, China. E-mail: dlyhy@dicp.ac.cn

${ }^{b}$ CAS Key Laboratory of Coastal Environmental Processes and Ecological Remediation, Yantai Institute of Coastal Zone Research, Chinese Academy of Sciences, Yantai 264003, China. E-mail: liangweizhang@yic.ac.cn, lxchen@yic.ac.cn

${ }^{c}$ College of Chemistry and Chemical Engineering, Yantai University, Yantai 264005, China

${ }^{d}$ Center for Ocean Mega-Science, Chinese Academy of Sciences, Qingdao 266071, China

$\dagger$ Electronic supplementary information (ESI) available. See DOI: 10.1039/ d1cc01110c
}

caused many diseases, such as slow growth in children, liver damage, ischemic stroke, hair pigmentation, edema, lethargy, skin lesions and weakness, muscle and fat loss, etc. ${ }^{7}$ Due to a similar structure and sensitivity to the environment of biological thiols, it remains a great challenge to develop novel tools for their discriminative and dynamic detection.

The traditional detection methods adopted for Cys analysis mainly include high performance liquid chromatography (HPLC) and capillary electrophoresis (CE). These methods can provide super resolution for Cys detection but required high-cost instruments, complicated pre-processing, long inspection time and other limitations. ${ }^{8}$ A critical fact is that the aforementioned methods cannot accomplish the real-time monitoring of species in living systems, which is especially important for environmentally sensitive species. Fluorescence imaging technology has gradually entered into the field of view of researchers due to its advantages of non-invasiveness, high specificity, visualization and real-time dynamic monitoring. And numerous fluorescent probes have been widely used in the imaging of biologically reactive species to reveal life phenomena in cells or in vivo in recent years, ${ }^{6-11}$ including related research on cysteine monitoring. A series of receptors were selected and used as recognition groups for probe design, such as 2,4-dinitrobenzenesulfonyl, ${ }^{12-16}$ acryloyl, ${ }^{17-23} \quad \alpha, \beta$-unsaturated ketone $^{24}$ nitrobenzodioxazole (NBD) and others, ${ }^{25-27}$ and we have summarized and compared the differences among them (Fig. 1A and Table S1, ESI $\dagger$ ). Although so many fluorescent probes have been developed for the detection of Cys, the current probes suffered from one way or another inadequacy (biocompatibility, selectivity and sensitivity), which greatly limit their application during the practical process. Furthermore, most of studies focus on novel probe design, rare researches have considered used them for real-time monitoring the stress response cysteine fluctuations to reveal its roles in living system. Therefore, the development of a novel imaging strategy to provide visual evidence for the stress response Cys fluctuations is needed.

Taking all these factors into account, we intend to design and synthesise a cysteine-selective fluorescent probe to monitor Cys fluctuations and provide visual evidence for the stress 


\section{(A) Commonly used recognition group}
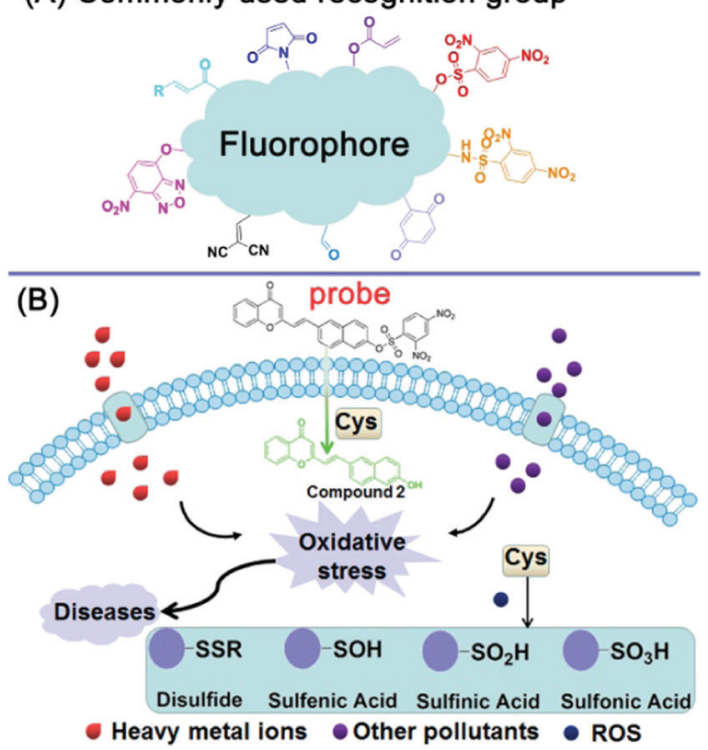

Fig. 1 (A) Commonly used recognition group for probe design in previous work. (B) The designed probe for monitoring the stress response cysteine fluctuations.

response Cys fluctuations in living systems. In recent years, our lab has been endeavoring to develop a series of fluorescent probes for the detection of endogenous biological species. ${ }^{28-31}$ We have developed a chemical covalent tactic for bio-thiol sensing using compounds containing a methyl sulfoxide moiety and accomplished protein thiol labeling. ${ }^{32}$ Also, we have successfully evaluated the protective effects of mitochondrial GSH during cerebral ischemia/reperfusion injury using a near-infrared GSH fluorescent probe. ${ }^{33}$ In this work, we selected compound 2 (Fig. 1B) as a fluorophore because its skeleton is suitable for ratiometric fluorescent probe design, which was already discovered in our previous work. ${ }^{32} 2,4$ Dinitrobenzenesulfonyl was selected as a recognition group because of its two characteristics: one is that it is a strong electron-deficient group and can effectively quench the fluorescence of fluorophores; the other is that it can be replaced by a strong nucleophile, which may provide a potential possibility of discriminatively detecting biological thiols relaying on their ionization constants ( $\mathrm{p} K_{\mathrm{a}}$, Cys, 8.3; GSH, 8.8; Hcy, 10.0). Thus, as expected, we have successfully designed and synthesized fluorescent probe 1 to detect Cys with high sensitivity and selectivity over GSH and Hcy. It can be used for imaging endogenous and exogenous cysteine in living cells. More importantly, we also successfully monitored Cys fluctuations and confirmed the relationship between the intracellular Cys level and oxidative stress through $\mathrm{Hg}^{2+}$ and $\mathrm{H}_{2} \mathrm{O}_{2}$ stimulations (Fig. 1B).

The probe was synthesized in two steps and the detailed route is shown in Scheme S1 (ESI $\dagger$ ). Firstly, the fluorophore (compound 2) was obtained by the coupled reaction of methyl and aldehyde groups according to our previously reported method. ${ }^{33}$ Subsequently, it reacted with 2,4-dinitrobenzenesulfonyl chloride under
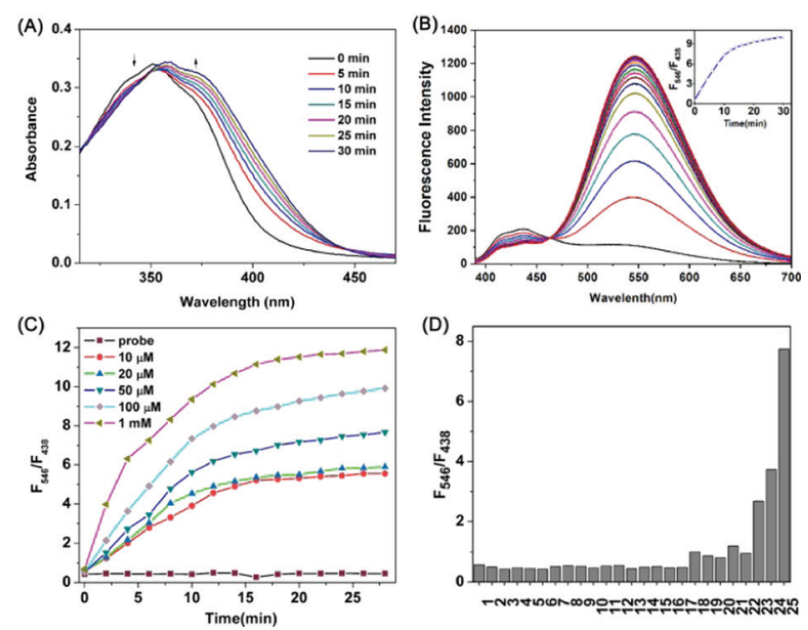

Fig. 2 The spectral studies of the probe in HEPES-EtOH buffer $(3 / 2, \mathrm{v} / \mathrm{v}$, $\mathrm{pH}=7.40)$. (A) Time-dependent absorption spectra of probe $1(10 \mu \mathrm{M})$ towards Cys $(100 \mu \mathrm{M}),(\mathrm{B})$ time-dependent fluorescence spectra of probe $1(10 \mu \mathrm{M})$ towards Cys $(100 \mu \mathrm{M}),(\mathrm{C})$ time- and dose-dependent fluorescent response of probe $1(10 \mu \mathrm{M})$ towards Cys $(0-1 \mathrm{mM})$, and (D) probe 1 in the presence of various analytes (100 $\mathrm{MM})$. 1-25 represent probe, $\mathrm{Mg}^{2+}, \mathrm{Na}^{+}$, $\mathrm{K}^{+}, \mathrm{Ca}^{2+}{ }^{2+} \mathrm{NO}_{3}{ }^{-}, \mathrm{Tyr}, \mathrm{L}-$ ascorbic acid, Asp, His, Val, Pro, Ala, Phe, Ser, Gly, Thr, GSSG, $\mathrm{ClO}^{-}$, cystine, $\mathrm{Hg}^{2+}, \mathrm{H}_{2} \mathrm{O}_{2}(500 \mu \mathrm{M}), \mathrm{Hcy}, \mathrm{GSH}$, and Cys.

organic bases to give probe 1 . All compounds were characterized using NMR and MS spectra in Fig. S1-S6 (ESI $\dagger$ ). With the probe in hand, we set out to determine its spectral properties. Probe 1 $(10 \mu \mathrm{M})$ and Cys $(100 \mu \mathrm{M})$ were measured in HEPES-EtOH buffer $(3 / 2, \mathrm{v} / \mathrm{v}, \mathrm{pH}=7.40)$. As can be seen from Fig. $2 \mathrm{~A}$, probe 1 showed the main absorption band at $350 \mathrm{~nm}$, and then shifted to $375 \mathrm{~nm}$ after Cys was added to the solution as time increased. In fluorescence spectra (Fig. 2B), probe 1 has weak blue fluorescence and the maximum emission wavelength center at $438 \mathrm{~nm}$. When Cys was added into the solution of probe 1 , the fluorescence at $438 \mathrm{~nm}$ gradually decreased, meanwhile the fluorescence at $546 \mathrm{~nm}$ increased rapidly within $10 \mathrm{~min}$, and then saturated slowly. The time- and dose-dependent fluorescent response of probe 1 towards Cys was also investigated (Fig. 2C). We further determined that the probe has a superior detection limit of up to $2.89 \mathrm{nM}(3 \sigma / k)$ for Cys (Fig. S7, ESI $\dagger$ ), ${ }^{34}$ which is lower than those of many reported probes (Table S1, ESI $\dagger$ ). The response mechanism was confirmed by HPLC analysis and HRMS (Fig. S8, S9 and Scheme S2, ESI $\dagger$ ). And the fluorophore displayed aggregation-induced emission character (Fig. S10, ESI $\dagger$ ). ${ }^{35}$ The biological environment is commonly complex, so it is important and necessary for the probe to have good selectivity. In order to confirm the selectivity and specificity of probe 1 for Cys, the potential interferent was investigated, including some biological thiols (GSH and Hcy), anions, cations $\left(\mathrm{Na}^{+}, \mathrm{K}^{+}, \mathrm{Ca}^{2+}, \mathrm{Mg}^{2+}\right.$, and $\left.\mathrm{Hg}^{2+}\right)$, some amino acids (Tyr, $\mathrm{L}^{-}$ ascorbic acid, Asp, His, Val, Pro, Ala, Phe, Ser, Gly, and Thr), and ROS $\left(\mathrm{H}_{2} \mathrm{O}_{2}\right.$ and $\left.\mathrm{ClO}^{-}\right)$. As shown in Fig. 2D, both GSH and Hcy elicited a certain enhancement much less than that of Cys a $F_{546} / F_{438}$ ratio, while others did not cause any fluorescence changes. Considering high concentrations of $\mathrm{Na}^{+}$and $\mathrm{K}^{+}$, we investigated the fluorescent response of the probe towards them. As shown in Fig. S11 (ESI $\dagger$ ), they did not affect Cys detection. 
These results proved that our probe 1 has high sensitivity and selectivity for the detection of Cys.

In light of the above excellent spectral studies, we wonder whether probe 1 has practical application in imaging in living cells. Before the imaging experiments, the MTT assays were carried out using Hep G2 cells. The cells have a high survival rate (more than $90 \%)$ in the presence of a probe $(0-50 \mu \mathrm{M}$, Fig. $\mathrm{S} 12, \mathrm{ESI} \dagger)$. It is worth mentioning that the survival rate of the cells was more than $99 \%$ when the concentration of probe 1 was $10 \mu \mathrm{M}$, suggesting that the probe has not caused great damage to the cells with low toxicity, and can be applied in cell experiments.

To evaluate the ability of the probe to monitor Cys in living cells, we applied it in the imaging of endogenous Cys using Hep G2 cells. The cells were incubated with the probe and observed for 5, 15, 20, and $30 \mathrm{~min}$, respectively. As shown in Fig. 3, green fluorescence signals gradually appeared and became strong with time. The probe is mainly distributed in the cytoplasm but the nucleus (Fig. S13, ESI $\dagger$ ). The probe was also successfully applied in imaging Cys in other cell lines, including A549, SHSY5Y and HL-7702 (Fig. S14, ESI $\dagger$ ). The results indicated that our probe can be used for the detection of endogenous Cys in living cells.

In order to further prove that our probe can be used for monitoring endogenous Cys fluctuations, we have performed a series of imaging experiments. Exogenous Cys was introduced into living cells by pretreatment with Cys for $30 \mathrm{~min}$, and the cells were further incubated with the probe for another $30 \mathrm{~min}$. As shown in Fig. 4f and g, the green fluorescence signals obviously become stronger due to elevation of the Cys level by exogenous addition. To impoverish the amount of Cys, a thiol scavenger $N$-ethylmaleimide (NEM) was selected for blocking them. The Hep G2 cells were incubated with NEM for $30 \mathrm{~min}$ and then with the probe for $30 \mathrm{~min}$. Confocal imaging results showed that green fluorescence was significantly weakened and nearly disappeared after NEM treatment (Fig. 4h). The fluorescence can be restored by the subsequent exogenous addition of cysteine (Fig. 4i). These results firmly confirmed that our probe is a practical tool for monitoring Cys fluctuations. In a living system, cysteine existed in the form of an independent individual or protein cysteine (including RSH and RSSR'). ${ }^{36}$ Under oxidative stress, RSH can be oxidized and transformed into disulfide. In order to understand protein cysteine fluctuations, we used NEM to block thiols and then treated with tris(2carboxyethyl)-phosphine (TCEP, a reducing agent commonly

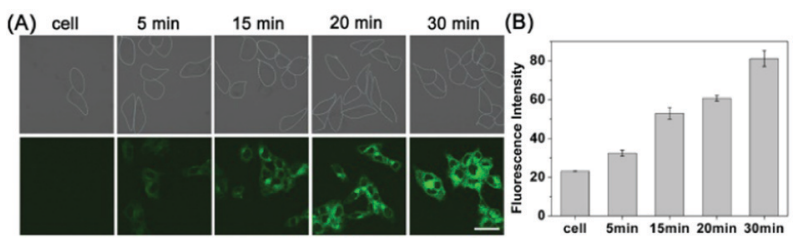

Fig. 3 (A) Time-dependent imaging of endogenous cysteine in Hep G2 cells using probe $1(10 \mu \mathrm{M})$, scale bars: $20 \mu \mathrm{m}$. (B) Fluorescence intensity was quantified by ImageJ.

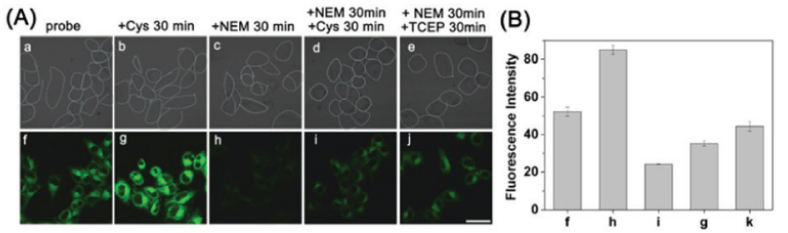

Fig. 4 (A) Imaging of Cys fluctuations in living cells using probe 1 . The cells were incubated with probe 1 for $30 \mathrm{~min}$ (a and f). The cells were pretreated with exogenous Cys $(100 \mu \mathrm{M})$ before incubation with the probe $(10 \mu M)(b$ and g). The cells were pretreated with NEM $(100 \mu M)$ before incubation with the probe $(10 \mu \mathrm{M})$ ( $c$ and h). The cells were sequentially incubated with NEM, Cys and the probe ( $d$ and i). The cells were sequentially incubated with NEM, TCEP and the probe (e and j). $\lambda_{\text {ex }}=440 \mathrm{~nm}$, $\lambda_{\mathrm{em}}=500-600 \mathrm{~nm}$. Scale bars: $20 \mu \mathrm{m}$. (B) Fluorescence intensity was quantified by ImageJ.

used for cleavage of disulfide in a biological system, $1 \mathrm{mM}$ ) for $30 \mathrm{~min}$. After the cells were incubated with the probe, green fluorescence was observed in the channels (Fig. 4j). In this part, we has successfully performed imaging experiments to regulate cysteine level by using NEM and TCEP for evaluation the practical application of probe in monitoring Cys fluctuations.

Heavy metal ions have attracted wide interest due to their deleterious effects on environmental security and public health, such as $\mathrm{Hg}^{2+}$ and $\mathrm{Cd}^{2+} \cdot{ }^{37}$ These metal ions easily accumulated in the body through the food chain and eventually lead to a number of serious diseases. However, there are rare research studies providing visual evidence of how heavy metal ions affect endogenous biological molecule fluctuations. Considering the high affinity of $\mathrm{Hg}^{2+}$ to sulfur, we intend to explore the relationship between $\mathrm{Hg}^{2+}$ and cysteine levels. As shown in Fig. 5A, the low concentration of $\mathrm{Hg}^{2+}$ did not cause obvious fluorescence change (less than $20 \mu \mathrm{M}$ ). The fluorescence was greatly quenched when the concentration of $\mathrm{Hg}^{2+}$ reached up to $50 \mu \mathrm{M}$, suggesting that the cysteine level was regulated by $\mathrm{Hg}^{2+}$. These results are mainly due to two factors, one is that the coordination of $\mathrm{Hg}^{2+}$ with cysteine (including the protein

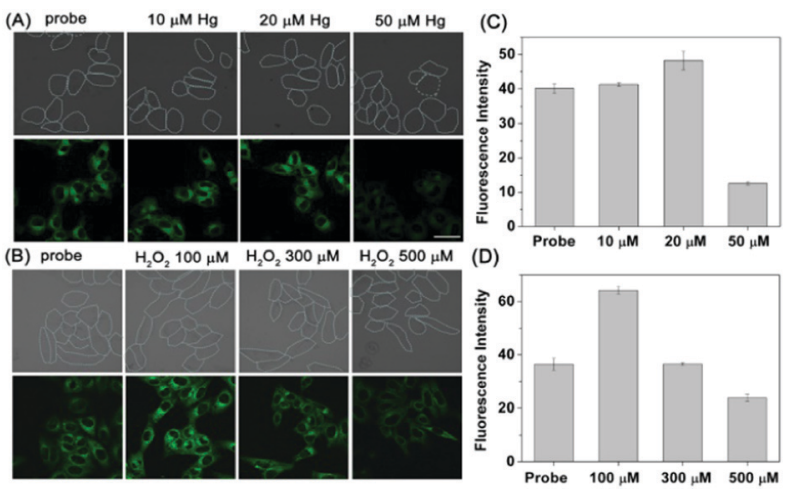

Fig. 5 Imaging of the stress response cysteine fluctuations using the probe. (A) The Hep G2 cells were incubated with different concentrations of $\mathrm{Hg}^{2+}(0,10,20$, and $50 \mu \mathrm{M})$ for $15 \mathrm{~min}$ before the addition of the probe. (B) The Hep G2 cells were incubated with different concentrations of $\mathrm{H}_{2} \mathrm{O}_{2}$ $(0,100,300$, and $500 \mu \mathrm{M})$ for $30 \mathrm{~min}$ before the addition of the probe. Scale bars: $20 \mu \mathrm{m}$. ( $C$ and D) Fluorescence intensity was quantified by ImageJ. 
cysteine residue) directly decreased the cysteine concentration; the other is that oxidative stress burst caused by coordination of $\mathrm{Hg}^{2+}$ reduces the antioxidant capacity of the system, which further decreased the cysteine concentration. $\mathrm{Hg}^{2+}$ could induce oxidative stress burst which has been confirmed in our previous work. ${ }^{38,39}$ To clarify the $\mathrm{Hg}^{2+}$ regulated cysteine level, we carried out imaging experiments to monitor cysteine fluctuations by pretreatment with different concentrations of $\mathrm{H}_{2} \mathrm{O}_{2}$. As shown in Fig. 5B, the fluorescence enhanced slightly

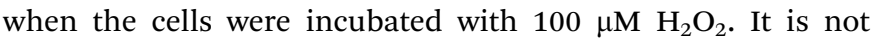
surprising that the cells will promote the biosynthesis of cysteine against oxidative stress when within the regulatory capacity of biological antioxidant systems. When the concentrations of $\mathrm{H}_{2} \mathrm{O}_{2}$ increased (more than $300 \mu \mathrm{M}$ ) and exceed self-regulation, the cysteine will be consumed leading to fluorescence decay. The fluorescent change owed to the regulated Cys fluctuation rather than cell apoptosis and confirmed via cytometric apoptosis assay using flow cytometry analysis, Hoechst 33342 and CCK-8 kit (Fig. S15-S17, ESI†). These results indicated that the probe can be used to monitor $\mathrm{Hg}^{2+}$ induced cysteine fluctuations in living cells, providing visual evidence of $\mathrm{Hg}^{2+}$ regulated cysteine fluctuations for the first time, which may open a new way to help researchers to reveal the mechanism of heavy metal ion poisoning.

In summary, we have successfully designed and synthesized a cysteine-selective fluorescent probe 1 to monitor Cys fluctuations. The solid spectral and imaging results demonstrated that probe 1 has excellent performance in monitoring endogenous and exogenous cysteine. The detection limit of the probe for Cys can reach $2.89 \mathrm{nM}$. Finally, we used the probe to monitor $\mathrm{Hg}^{2+}$ induced cysteine fluctuations in living cells, providing visual evidence of $\mathrm{Hg}^{2+}$ regulated cysteine fluctuations for the first time, confirming the relationship between the intracellular Cys level and oxidative stress caused by $\mathrm{Hg}^{2+}$. This work may open a new way to help researchers to reveal the mechanism of heavy metal ion poisoning and provide insights into the roles of Cys in future research.

This work was financially supported by the Natural Science Foundation of China (21778026, 21701074, 21402077 and 21976209), the Program of Youth Innovation Promotion Association, CAS (2019217) and the Taishan Scholar Project Special Funding (TS20190962).

\section{Conflicts of interest}

There are no conflicts to declare.

\section{Notes and references}

1 L. B. Poole, Free Radical Biol. Med., 2015, 80, 148-157.

2 M. T. Goodman, K. McDuffie, B. Hernandez, L. R. Wilkens and J. Selhub, Cancer, 2000, 89, 376-382.

3 W. H. Watson, J. D. Ritzenthaler, P. Peyrani, T. L. Wiemken, S. Furmanek, A. M. Reyes Vega, T. J. Burke, Y. Zheng, J. A. Ramirez and J. Roman, Free Radical Biol. Med., 2019, 143, 55-61.
4 G. J. McBean, M. Aslan, H. R. Griffiths and R. C. Torrao, Redox Biol., 2015, 5, 186-194.

5 B. D. Paul, J. I. Sbodio and S. H. Snyder, Trends Pharmacol. Sci., 2018, 39, 513-524.

6 G. Yin, T. Niu, T. Yu, Y. Gan, X. Sun, P. Yin, H. Chen, Y. Zhang, H. Li and S. Yao, Angew. Chem., Int. Ed., 2019, 58, 4557-4561.

7 X. Chen, Y. Zhou, X. Peng and J. Yoon, Chem. Soc. Rev., 2010, 39, 2120-2135.

8 L. Y. Niu, Y. Z. Chen, H. R. Zheng, L. Z. Wu, C. H. Tung and Q. Z. Yang, Chem. Soc. Rev., 2015, 44, 6143-6160.

9 D. Cao, Z. Liu, P. Verwilst, S. Koo, P. Jangjili, J. S. Kim and W. Lin, Chem. Rev., 2019, 119, 10403-10519.

10 J. Liu, M. Liu, H. Zhang, X. Wei, J. Wang, M. Xian and W. Guo, Chem. Sci., 2019, 10, 10065-10071.

11 X. Wang, J. Zha, W. Zhang, W. Zhang and B. Tang, Analyst, 2020, 145, 6119-6124.

12 W. Du, R.-J. Liu, J. Fang, H. Gao, Y.-W. Wang and Y. Peng, Tetrahedron, 2019, 75, 130477.

13 D. Chen, Z. Long, Y. Sun, Z. Luo and X. Lou, J. Photochem. Photobiol., A, 2019, 368, 90-96.

14 L. Yang, H. Xiong, Y. Su, H. Tian, X. Liu and X. Song, Chin. Chem. Lett., 2019, 30, 563-565.

15 M. Wei, P. Yin, Y. Shen, L. Zhang, J. Deng, S. Xue, H. Li, B. Guo, Y. Zhang and S. Yao, Chem. Commun., 2013, 49, 4640.

16 L. Fan, W. Zhang, X. Wang, W. Dong, Y. Tong, C. Dong and S. Shuang, Analyst, 2019, 144, 439-447.

17 Y. Liu, Y. X. Wu, D. Zhang, H. Zhong, D. Li, K. He, W. T. Wei and S. Yu, Talanta, 2020, 220, 121364.

18 Y. Gan, G. Yin, T. Yu, Y. Zhang, H. Li and P. Yin, Talanta, 2020, 210, 120612.

19 X. Hou, Z. Li, Y. Li, Q. Zhou, C. Liu, D. Fan, J. Wang, R. Xu and Z. Xu, Spectrochim. Acta, Part A, 2021, 246, 119030.

20 L.-F. Wei, N. Thirumalaivasan, Y.-C. Liao and S.-P. Wu, Spectrochim. Acta, Part A, 2017, 183, 204-208.

21 Y. Zhang, Y. Zhang, Y. Yue, J. Chao, F. Huo and C. Yin, Sens. Actuators, B, 2020, 320, 128348.

22 M. Qian, J. Xia, L. Zhang, Q. Chen, J. Guo, H. Cui, Y. S. Kafuti, J. Wang and X. Peng, Sens. Actuators, B, 2020, 321, 128441.

23 P. Xu, M. Liu, T. Gao, H. Zhang, Z. Li, X. Huang and W. Zeng, Tetrahedron Lett., 2015, 56, 4007-4010.

24 Z. Guo, S. Nam, S. Park and J. Yoon, Chem. Sci., 2012, 3, 2760.

25 C. Ge, F. Shen, Y. Yin, K. Chang, X. Zhang, P. Zhou, J. Li, Y. Liu and C. Lu, Talanta, 2021, 223, 121758.

26 J. Hong and G. Feng, Sens. Actuators, B, 2021, 326, 129016.

27 X. Zhang, C. Liu, X. Cai, B. Tian, H. Zhu, Y. Chen, W. Sheng, P. Jia, Z. Li, Y. Yu, S. Huang and B. Zhu, Sens. Actuators, B, 2020, 310, 127820.

28 L. Zhang, S. Peng, J. Sun, R. Liu, S. Liu and J. Fang, Chem. Commun., 2019, 55, 1502-1505.

29 L. Zhang, D. Duan, Y. Liu, C. Ge, X. Cui, J. Sun and J. Fang, J. Am. Chem. Soc., 2014, 136, 226-233.

30 L. Zhang, S. Peng, J. Sun, J. Yao, J. Kang, Y. Hu and J. Fang, Chem. Sci., 2017, 8, 2966-2972.

31 L. Zhang, X. Cui, J. Sun, Y. Wang, W. Li and J. Fang, Bioorg. Med. Chem. Lett., 2013, 23, 3511-3514.

32 L. W. Zhang, J. Kang, S. D. Liu, X. Zhang, J. Y. Sun, Y. S. Hu, Y. Yang and L. X. Chen, Chem. Commun., 2020, 56, 11485-11488.

33 X. Zhang, Y. Huang, X. Han, Y. Wang, L. Zhang and L. Chen, Anal. Chem., 2019, 91, 14728-14736.

34 G. H. Aryal, K. Lu, G. Chen, K. W. Hunter and L. Huang, Chem. Commun., 2019, 55, 13912-13915.

35 G. H. Aryal, G. R. Rana, F. Guo, K. W. Hunter and L. Huang, Chem. Commun., 2020, 56, 13437-13440.

36 G. Hu, H. Jia, L. Zhao, D.-H. Cho and J. Fang, Chin. Chem. Lett., 2019, 30, 1704-1716.

37 T. L. Hwang, H. Y. Chen, T. T. Changchien, C. C. Wang and C. M. Wu, Biomed. Rep., 2013, 1, 379-382.

38 Y. Wang, L. Zhang and L. Chen, Anal. Chem., 2020, 92, 1997-2004.

39 Y. Wang, L. Zhang, S. Zhang, Z. Liu and L. Chen, Anal. Chem., 2019, 91, 7812-7818. 